\title{
ANÁLISIS DE CALIDAD DE LOS RECURSOS ESTADÍSTICOS (SECCIÓN MATERIAL Y MÉTODO) EN LOS PROYECTOS DE TESIS DE UN DEPARTAMENTO UNIVERSITARIO
}

\author{
Ó. REGOJO ZAPATA*, F. LAMATA HERNÁNDEZ**, J.M. SÁNCHEZ ZALABARDO*, \\ Á. ELIZALDE BENITO*, J. NAVARRO GIL*, J.G. VALDIVIA URÍA*
}

*Servicio de Urología. **Servicio de Cirugía General. Hospital Clínico Universitario Lozano Blesa. Zaragoza. Departamento de Cirugía de la Universidad de Zaragoza.

Actas Urol Esp. 28 (8): 581-587, 2004

\section{RESUMEN}

ANÁLISIS DE CALIDAD DE LOS RECURSOS ESTADÍSTICOS (SECCIÓN MATERIAL Y MÉTODO) EN LOS PROYECTOS DE TESIS DE UN DEPARTAMENTO UNIVERSITARIO

INTRODUCCIÓN Y OBJETIVOS: Los estudios sobre la calidad de tesis y proyectos de investigación en ciencias biomédicas son muy escasos, pero de enorme interés para la docencia universitaria, por la necesidad académica de mejorar la calidad del proceso de elaboración de la tesis. Los objetivos del estudio fueron determinar la calidad de los proyectos de tesis de nuestro departamento, según el cumplimiento de la metodología científica y establecer, si existe, una relación entre la calidad global del proyecto y los recursos estadísticos utilizados.

MATERIAL Y MÉTODO: Estudio descriptivo en el que se analizaron 273 proyectos de tesis realizados entre 1995 y 2002 en el departamento de Cirugía de la Universidad de Zaragoza. La revisión se realizó por 15 observadores que analizaron 28 indicadores de cada proyecto. Dando un valor ponderado a cada uno de los indicadores, se calificaron los proyectos en una escala de 0 a 10 según la calidad en el cumplimiento de la metodología científica.

RESULTADOS: La media de las calificaciones de los proyectos fue de 5,53 (D.E: 1,77.) Un 13,9\% de los proyectos de tesis se concluyeron con la defensa y lectura del trabajo. En los tres indicadores de recursos estadísticos se encontró una diferencia estadísticamente significativa con la valoración de la calidad de los proyectos.

DISCUSIÓN: La calidad de los recursos estadísticos es de suma importancia cuando se quiere realizar un proyecto de tesis con buena metodología, ya que asegura llegar a conclusiones ciertas. En nuestro estudio hemos encontrado que más de la tercera parte de la variabilidad en la calidad del proyecto de tesis se explica por los tres ítems estadísticos referidos.

PALABRAS CLAVE: Estudio de calidad. Proyectos de tesis. Estadística.

\section{ABSTRACT}

QUALITY ANALISIS OF THE STADISTICAL USED RESOURCES (MATERIAL AND METHODS SECCION) IN THESIS PROJECTS OF A UNIVERSITARY DEPARTMENT

INTRODUCTION AND OBJETIVES: Studies about quality in thesis and investigation projects in biomedical sciences are unusual, but very important in university teaching because is necessary to improve the quality elaboration of the thesis. The objectives the study were to determine the project's quality of thesis in our department, according to the fulfillment of the scientific methodology and to establish, if it exists, a relation between the global quality of the project and the statistical used resources.

MATERIAL AND METHODS: Descriptive study of 273 thesis projects performed between 1995-2002 in surgery department of the Zaragoza University. The review realized for 15 observers that they analyzed 28 indicators of every project. Giving a value to each of the indicators, the projects qualified in a scale from 1 to 10 according to the quality in the fulfillment of the scientific methodology.

RESULTS: The mean of the project's quality was 5.53 (D.E: 1.77). In 13.9\% the thesis projects was concluded with the reading of the work. The three indicators of stadistical used resources had a significal difference with the value of the quality projects.

DISCUSSION: The quality of the statistical resources is very important when a project of thesis wants to be realized by good methodology, because it assures to come to certain conclusions. In our study we have thought that more of the third part of the variability in the quality of the project of thesis explains for three statistical above-mentioned articles.

KEY WORDS: Quality study. Thesis project. Stadistical. 
$\mathrm{U}$ na forma de evaluar la calidad de la investigación en una universidad es conocer la creación de nuevos conocimientos que se generan en ella. Estos conocimientos están presentes en las tesis doctorales y en los proyectos de investigación que se desarrollan en la universidad a lo largo del curso académico.

Los estudios sobre la calidad de tesis y proyectos de investigación en ciencias biomédicas son muy escasos ${ }^{1}$, pero de enorme interés para la docencia universitaria, pues permiten observar deficiencias metodológicas en los trabajos de investigación para subsanarlas en la tarea docente de post-grado.

La utilidad de este tipo de estudios proviene de la necesidad académica de mejorar la calidad del proceso de elaboración de la tesis. Una baja calidad en el proceso de tesis puede dañar de forma seria la calidad general de la gestión académica institucional. Así la investigación universitaria con problemas de calidad no cumple con su función de ser un bien de capital para la educación. A la vez esas deficiencias provocan una débil remuneración social, cognoscitiva y financiera, lo cual genera un menor prestigio institucional $^{2}$.

La solución a estos problemas pasa por un perfeccionamiento de la investigación universitaria y dentro de ella, del proceso de elaboración de la tesis. Pero ello requiere de una evaluación del estado actual y de los factores que contribuyen a esa situación de calidad en el presente ${ }^{3}$. El conocimiento de los factores de calidad en las tesis es un paso previo al establecimiento de todo un sistema de acciones encaminadas a mejorar la calidad, tales como la reformulación de guías para la elaboración de tesis, la capacitación de los profesores, tutores y lectores, la reglamentación del control de calidad de las tesis y la exigencia de un control de calidad en los actos de defensa de tesis ${ }^{2}$.

Los objetivos de este estudio fueron:

1. Determinar la calidad de los proyectos de tesis realizados en el departamento de cirugía de la facultad de medicina de la universidad de Zaragoza desde el año 1995, según el cumplimiento de la metodología científica.

2. Establecer, si existe, una relación entre la calidad global del proyecto y tres items del mate- rial y método de los estudios: variable predictora y de desenlace, test estadísticos utilizados y cálculo del tamaño muestral.

3. Establecer una nueva variable que agruparía los tres items antes mencionados, para establecer en cada proyecto la "calidad de los recursos estadísticos utilizados".

Proponemos como hipótesis del estudio, 1. la existencia de una relación estadísticamente significativa entre la calidad global de los proyectos y la existencia de una variable predictora, la enumeración de los test estadísticos utilizados y el cálculo del tamaño muestral. 2. Así mismo proponemos que la nueva variable llamada "calidad de recursos estadísticos utilizados" tiene una correlación directa con la calidad global de los proyectos.

\section{MATERIAL Y MÉTODO}

Se llevó a cabo una revisión de 273 proyectos de tesis presentados en el departamento de Cirugía, Obstetricia y Ginecología de la Facultad de Medicina de la Universidad de Zaragoza entre los años 1995 y 2002. La revisión se realizó por 15 observadores que analizaron 28 indicadores agrupados en 7 secciones: 1. Tema del proyecto de investigación. 2. Revisión de los conocimientos. 3. Hipótesis de trabajo. 4. Material y método. 5. Bibliografía. 6. Extensión. 7. Calificación global del proyecto. Cada uno de los proyectos fue revisado por al menos dos observadores para controlar la subjetividad de las calificaciones.

Los resultados obtenidos en la plantilla de cada proyecto se introdujeron en una base de datos estadística, realizándose el análisis con el programa Statview. Se utilizó el análisis de la varianza para la comparación de medias, el coeficiente de correlación de Pearson para conocer la correlación de dos variables numéricas y el coeficiente de determinación para conocer la utilidad del modelo establecido ${ }^{4-6}$.

La valoración se realizó por el análisis de los indicadores de calidad de los proyectos de tesis: diseño del experimento, sujetos, población, criterios de selección muestral, cumplimientos de normas éticas, métodos de asignación a grupos, descripción de tratamientos, materiales, métodos de control de calidad de los datos etc. Se analizó así mismo la bibliografía utilizada en cada pro- 
yecto, apuntando la correcta utilización de las normas Vancouver en las referencias, y la utilización de citas de los últimos cinco años asî como las publicaciones internacionales de impacto. Dando un valor ponderado a cada uno de los indicadores se calificaron los proyectos en una escala de 0 a 10 según la calidad obtenida en el cumplimiento de cada uno de los items (anexo 1), asignando 2 puntos al tema del proyecto (descripción y definición del problema, pertinencia y factibilidad del tema, definición de objetivos). 2 puntos a la revisión de los conocimientos (según su descripción, información, marco teórico perfil del problema y objetivos claros y realizables). 2 puntos a la hipótesis de trabajo sin ella, con defectos, hipótesis conceptual y operativa) y por último 4 puntos al material y métodos de estudio (diseño del experimento, sujeto, población, selección muestral, normas éticas, método de asignación del tratamiento, materiales utilizados, método de control de calidad de los datos, variables principales del estudio, test estadísticos utilizados y cálculo del tamaño muestral. El peso de estos tres últimos items en la valoración global del proyecto supone un $10 \%$, es decir, correctamente cumplimentados suman entre los tres un punto de los teóricamente 10 posibles. Pretendemos conocer si estos parámetros se correlacionan adecuadamente con la valoración global del proyecto (calidad del proyecto) o bien son independientes ${ }^{7}$.

Por otra parte, reseñar que el apartado bibliográfico, aunque se analizó y se estudió, no fue incluido en la valoración del proyecto debido a que las normas de nuestra universidad no lo fijan como obligatorio, y por ello a quienes no lo presentan no se les puede penalizar en la calidad global.

\section{RESULTADOS}

La valoración de los proyectos sigue una distribución normal con media de las calificaciones de 5,53 (D.E: 1,77). (Tabla 1 y Figura 1)

Un $13,9 \%$ de los proyectos de tesis concluyeron con la defensa y lectura del trabajo, pero no se ha encontrado que los proyectos de las tesis leídas fueran de más calidad que las no leídas. Hay que tener en cuenta que los proyectos
Tabla 1

Estadísticos descriptivos de la calidad de los proyectos de tesis

\begin{tabular}{lc}
\hline & Calidad \\
\hline Media & 5,529 \\
Std. Dev. & 1,774 \\
Std. Error &, 076 \\
Muestra & 541 \\
Mínimo &, 667 \\
Máximo & 9,991 \\
Perdidos & 5 \\
\hline
\end{tabular}

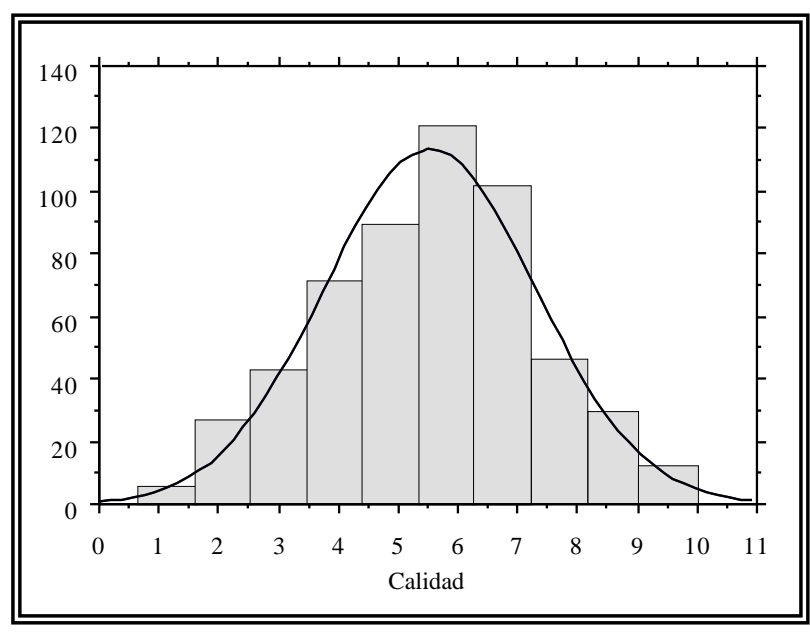

Figura 1. Histograma de la calidad de los proyectos de tesis.

presentados en los últimos años no han tenido tiempo para demostrar su concreción o no en una tesis.

Sin embargo en los tres indicadores de recursos estadísticos se encontró una diferencia estadísticamente significativa con la valoración de la calidad de los proyectos.

La media de la valoración global de los proyectos que no definían las variables predictoras y de desenlace era de 4,5 puntos, los proyectos que las definían de manera imprecisa tenían una media de 5,3 puntos, y la media de los proyectos que definían detalladamente estas variables era de 6,9 puntos, encontrándose una diferencia estadísticamente significativa entre estas medias para un $\mathrm{p}<0,001$. (Tabla 2 y Figura 2).

En lo referente a la mención de los test estadísticos en los proyectos, la media de la valoración global de los proyectos que no referían los 
Tabla 2

Análisis de la varianza para comparación de medias en el análisis de las variables de desenlace

\section{Variable de desenlace}

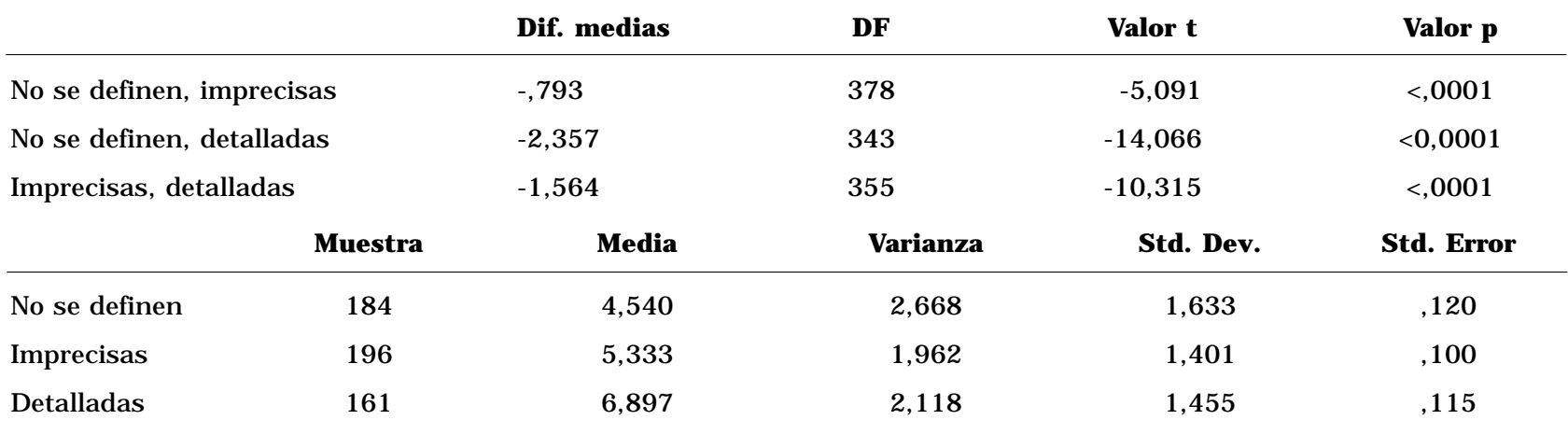

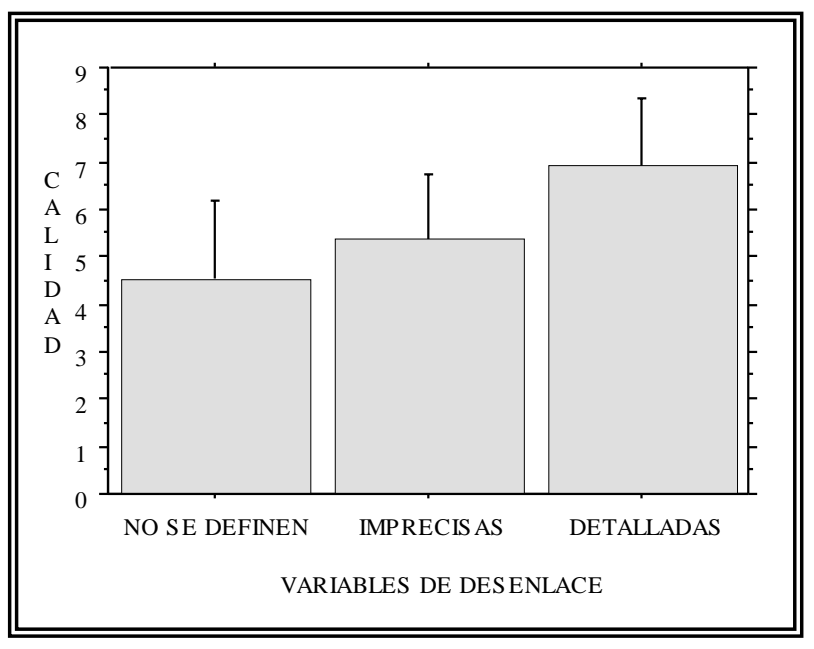

Figura 2. Comparación de medias de calidad de las tesis según la definición en el trabajo, de las variables de desenlace.

test estadísticos era de 4,9 puntos, los proyectos que los referian con errores o que sólo los enumeraban tenían una media de 5,5 puntos, y la media de los proyectos que detallaban y justificaban los test estadísticos era de 6,9 puntos, encontrándose una diferencia estadísticamente significativa entre estas medias para un $\mathrm{p}<0,001$ (Tabla 3 y Figura 3).

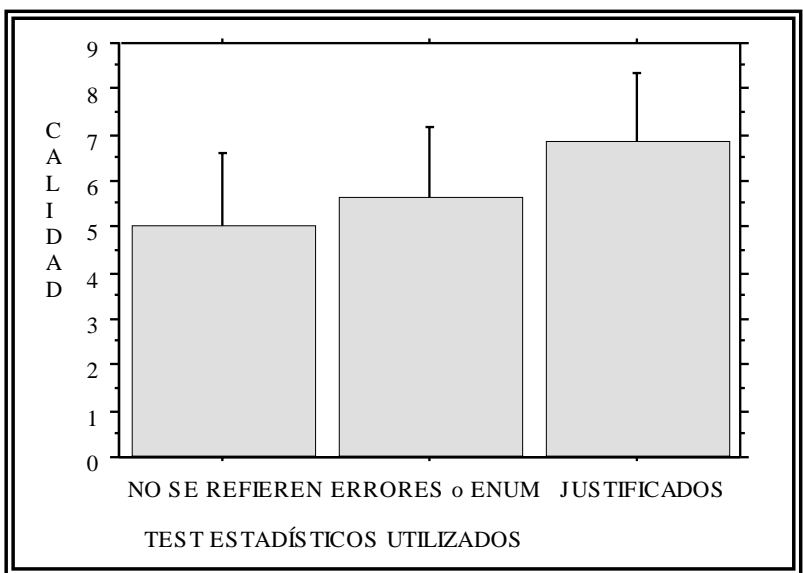

Figura 3. Comparación de medias de calidad de las tesis según la mención en el trabajo, de los test estadisticos utilizados.

Tabla 3

Análisis de la varianza para comparación de medias en el análisis de los test estadísticos utilizados

\section{Test estadísticos}

\begin{tabular}{lccccc} 
& & Dif. medias & DF & Valor t & Valor p \\
\hline No se refieren & &,- 611 & 427 & $-3,755$ &, 0002 \\
Errores o enum & & $-1,849$ & 368 & $-10,016$ & $<0,0001$ \\
Justificados & & $-1,238$ & 281 & $-6,355$ & $<, 0001$ \\
& Muestra & Media & Varianza & Std. Dev. & Std. Error \\
\hline No se refieren & 258 & 4,953 & 2,764 & 1,633 &, 104 \\
Errores o enum & 171 & 5,564 & 2,661 & 1,631 &, 125 \\
Justificados & 112 & 6,801 & 2,421 & 1,556 &, 147 \\
\hline
\end{tabular}


Por lo que se refiere al análisis de tamaño muestral, este indicador es el que establece mejor la relación con la calidad global de los proyectos, existiendo una diferencia de medias de 3,02 puntos entre los proyectos que no refieren el tamaño muestral y los que lo calculan correctamente. La media de la valoración global de los proyectos que no referían el tamaño muestral era de 4,9 puntos, los proyectos que lo refieren pero no dicen cómo se calculó tenían una media de 5,6 puntos, y la media de los proyectos calculaban el tamaño muestral correctamente era de 7,9 puntos, encontrándose una diferencia estadísticamente significativa entre estas medias para un p<0,001 (Tabla 4 y Figura 4).

La calidad de los recursos estadísticos utilizados es un nuevo indicador que aparece con la suma de los tres anteriores. En cada uno de los proyectos se dieron unos puntos de los tres indicadores antes mencionados:

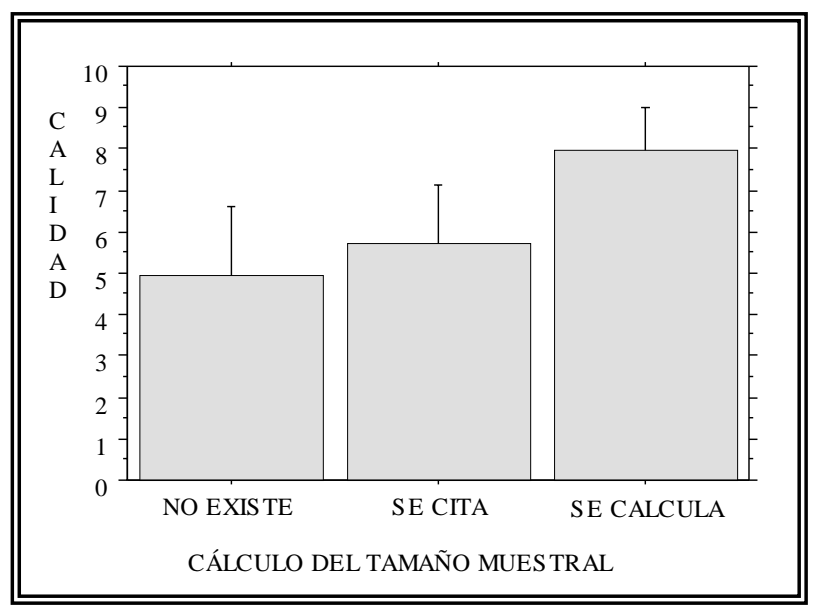

FIGURA 4. Comparación de medias de calidad de las tesis según la mención en el trabajo, del cálculo del tamaño muestral.
1. Variable predictora o de desenlace: a) No se define $(0,00)$; b) Imprecisas $(0,50)$; c) Detalladas $(1,00)$.

2. Test estadísticos: a) No se refieren $(0,00)$; b) Con errores o sólo se enumeran $(0,50)$; c) Detallados y justificados $(1,00)$.

3. Tamaño muestral: a) No existe $(0,00)$; b) Se refiere pero no cómo se calculó $(1,00)$; c) Se calcula correctamente $(2,00)$.

Así cada proyecto tomaba un valor de calidad de los recursos estadísticos, suma de los tres indicadores y este valor es el que se ha comparado con la calidad global del proyecto (Tabla $5 \mathrm{y}$ Figura 5).

Se ha encontrado que este nuevo indicador es el que presenta mejor correlación con la calidad del proyecto de tesis, encontrándose un coeficiente de correlación de 0,6 para un $\mathrm{p}<0,001$. El coeficiente de determinación $\mathrm{R}$ ? es de 0,38. O sea, la calidad de los recursos estadísticos es una variable que explica casi un $40 \%$ de la calidad de los proyectos (Tabla 6 y Figura 6).

\section{Tabla 5}

Estadísticos descriptivos de la calidad de los recursos estadísticos

Calidad de los recursos estadísticos

$\begin{array}{lc}\text { Media } & 1,486 \\ \text { Std. Dev. } & 1,052 \\ \text { Std. Error } & , 045 \\ \text { Muestra } & 546 \\ \text { Mínimo } & 0,000 \\ \text { Máximo } & 4,000 \\ \text { Perdidos } & 0\end{array}$

Perdidos

0

Tabla 4

Análisis de la varianza para comparación de medias en el análisis del cálculo del tamaño muestral

\begin{tabular}{lccccc}
\hline Tamaño muestral & & & & & \\
& & Dif. medias & DF & Valor t & Valor $\mathbf{p}$ \\
\hline No existe, se cita & &,- 759 & 488 & $-5,255$ & $<, 0001$ \\
No existe, se calcula & & $-3,010$ & 292 & $-11,948$ & $<0,0001$ \\
Se cita, se calcula & & $-2,252$ & 296 & $-10,363$ & $<, 0001$ \\
& Muestra & Media & Varianza & Std. Dev. & Std. Error \\
\hline No existe & 243 & 4,899 & 2,968 & 1,723 &, 111 \\
Se cita & 247 & 5,657 & 2,145 & 1,465 &, 093 \\
Se calcula & 51 & 7,909 & 1,260 & 1,123 &, 157
\end{tabular}




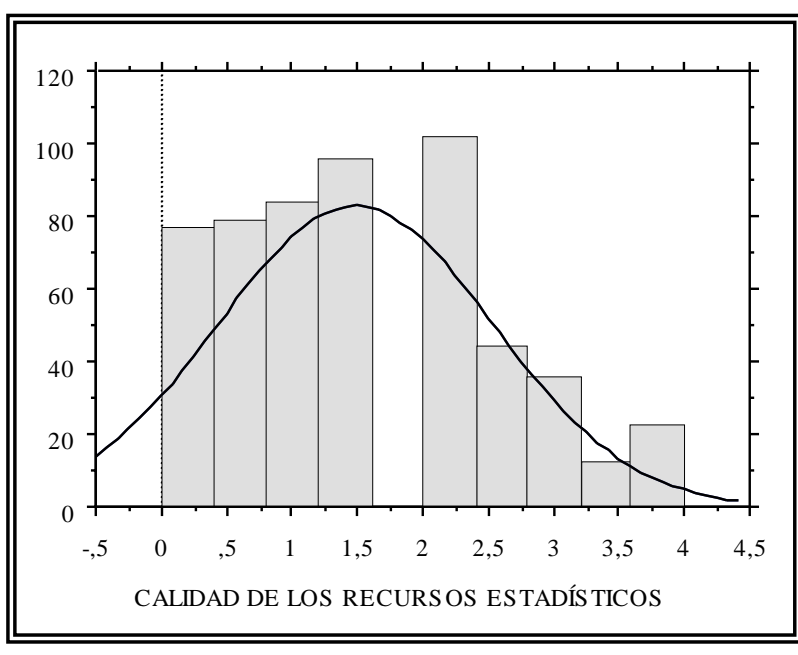

FIGURA 5. Histograma de la calidad de los recursos estadisticos.

Tabla 6

Coeficiente de correlación entre la calidad global de los proyectos y la calidad de sus recursos estadísticos

\section{Coeficiente de correlación}

Correlación Muestra Valor-z Valor-p

\begin{tabular}{lllll}
$\begin{array}{l}\text { Calidad, recursos } \\
\text { estadísticos }\end{array}$ &, 615 & 541 & 16,614 & $<, 0001$ \\
\hline
\end{tabular}

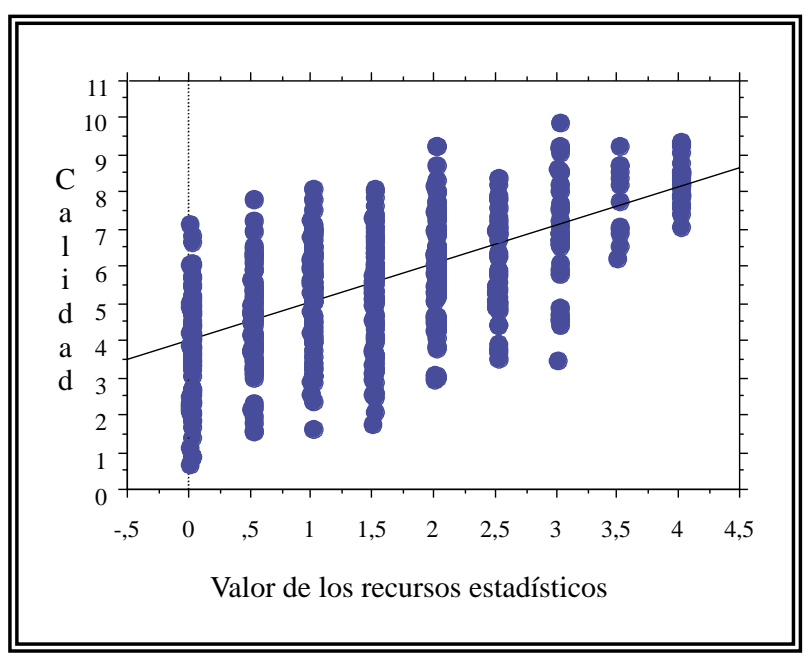

FIGURA 6. Diagrama de puntos que muestra la relación entre la calidad global de los proyectos y la calidad de sus recursos estadisticos.

\section{DISCUSIÓN}

A la hora de preparar un proyecto de tesis o de investigación es imprescindible hacer un buen planteamiento metodológico. Esto supone tener una buena bibliografía, realizar un buen resumen de los conocimientos que ya existen sobre el tema, plantear una o varias hipótesis y concretar el material y método del estudio. Dentro de todos los aspectos que hay que tener en cuenta para la creación de un proyecto, nos hemos centrado en los "recursos estadísticos". En nuestro estudio comprobamos que la puntuación media de la calidad de los proyectos era superior en los trabajos en que se mencionaba la variable predictora o de desenlace, en los trabajos en que se precisaban los test estadísticos utilizados y en los que se realizaba un buen cálculo del tamaño muestral. Esto nos recuerda que son aspectos que no hay que descuidar en la configuración del material y método del proyecto.

A la vez hemos creado un nuevo marcador capaz de mejorar la relación que los anteriores tenían con la calidad global del proyecto. La calidad de los recursos estadísticos es de suma importancia cuando se quiere realizar un proyecto de tesis con buena metodología, ya que asegura llegar a conclusiones ciertas. En nuestro estudio hemos encontrado que más de la tercera parte de la variabilidad en la calidad del proyecto de tesis se explica por los tres ítems estadísticos referidos.

Nuestro estudio no tenía la intención de comparar el papel que tienen otras variables en la calidad del proyecto de tesis. Nos hemos centrado en los recursos estadísticos, y con los datos estadísticos obtenidos podemos concluir:

\section{CONCLUSIONES}

1. Existe una relación estadística entre la calidad de los proyectos de tesis y la mención de la variable predictora de desenlace, la enumeración de los tests estadísticos utilizados y el cálculo del tamaño muestral.

2. Los recursos estadísticos en un proyecto de tesis, explican más de un tercio de la calidad del proyecto. Así pues parece importante insistir en este aspecto de la metodología si queremos elevar la calidad global de las tesis de nuestra universidad. 


\section{REFERENCIAS}

1. Bailar III JC, Mosteller F. Medical uses of statistics. Boston: N Engl J Med books, 1992.

2. Bland JM, Altman DG. Statistics notes: the use of transformations when comparing two means. BMJ 1996;12:1153.

3. Guallar E, Conde J, de la Cal M.A. Guía para la evaluación de proyectos de investigación en ciencias de la salud. Medicina Clínica 1997;108(12).

4. Armitage P. Interim analysis in clinical trials. Stat Med 1991;10:925-937.

5. Pértegas Díaz S, Pita Fernández S. Determinación del tamaño muestral para calcular la significación del coeficiente de correlación lineal. Cad Aten Primaria 2001-2002;9:209-211.

6. Pita Fernández S. Determinación del tamaño muestral. Cad Aten Primaria 1996;3:138-141.

7. Zamora MM, Estavillo J (2001): Modelo de regresión normal clásico, [en linea] 5campus.org, Econometría <http://www. 5campus.org/ leccion/ecoreg>
8. Roque Pujol R. Evaluación de calidad de tesis universitarias. www.geocities.com.

9. Exploración de la calidad de las tesis de enfermería de la Facultad de Medicina de San Fernando, Prof. Hernán Sanabria Rojas, Prof. Luz Bullón Camarena. Universidad Nacional Mayor de San Marcos. Facultad de Medicina de San Fernando. Departamento Académico de Medicina Preventiva y Salud Pública. wwwl.unam.mx/udual/ Revista/20/CalidadTesisEnfer.htm.

Dr. O. Regojo Zapata

Av. Camino de las Torres, $112-1^{\circ}$

50007 Zaragoza

(Trabajo recibido el 19 abril de 2004) 